\title{
No light at the end of the tunnel
}

\author{
H. R. Reiss ${ }^{1,2, a}$ (D) \\ 1 Max Born Institute, Berlin, Germany \\ 2 American University, Washington, DC, USA
}

Received 9 January 2021 / Accepted 28 April 2021 / Published online 18 May 2021 (C) The Author(s) 2021

\begin{abstract}
The tunneling model for laser-induced processes implies the replacement of the propagating field of a laser by an oscillatory electric field. The view of the electric field as the primary influence in charged particle interactions fails for laser processes where the propagation property is important. Electric fields lack several quintessential laser-field properties that become dominant at high intensities and/or low frequencies. Quantum tunneling is not a concept generally suited to laser light. Conversely, laser criteria do not apply to electric-field phenomena like Sauter-Schwinger pair production in the vacuum, contrary to a widespread assumption.
\end{abstract}

\section{Introduction}

The tunneling model of electromagnetically induced processes is appropriate for static and quasistatic electric fields, but not for laser fields. The tunneling model has produced useful results over a limited range of parameters, but it fails catastrophically as laser intensity increases or laser frequency decreases, leading to false physical inferences. The concept of quantum tunneling through a potential barrier dates from the explanation of nuclear alpha decay of heavy nuclei by Gamow [1], and the ionization of an atom by an electric field by Oppenheimer [2]. In both cases, the causative agent in the process is a static electric field. When a laser field is sufficiently intense that it is the dominant influence in an interaction, then the fact that laser fields propagate at the speed of light in vacuum becomes fundamental, and the tunneling model fails. The importance of a propagating field is determined by the ponderomotive potential of a charged particle in the field, not by the strength of the electric component [3]. The ponderomotive potential is proportional to field intensity and inversely proportional to the square of the field frequency. Thus, failure of the tunneling model arises from high intensity and/or low frequency, either of which can generate relativistic effects $[4-6]$.

In quantum electrodynamics (QED), perturbative coupling between electromagnetic fields and charged particles is measured by the fine-structure constant $\alpha$. When a transverse field is nonperturbatively intense, coupling is measured by the ponderomotive potential $U_{p}[3,7]$. With the dipole approximation (DA) as employed in the atomic, molecular and optical (AMO) community, the sole electromagnetic quantity that remains is the electric field $\mathbf{E}$, so coupling to the

\footnotetext{
a e-mail: reiss@american.edu (corresponding author)
}

field of a particle of charge $q$ is determined by $q \mathbf{E}$. This leads to the DA concept, inappropriate for intense laser fields, that the electric field is the ultimate determinant of electromagnetic phenomena.

Propagating fields like laser fields possess electric and magnetic fields that lie in a plane perpendicular (i.e., transverse) to the direction of propagation. Static or oscillatory electric fields are longitudinal. Transverse and longitudinal fields have fundamentally different properties, and the tunneling model applies only to longitudinal fields.

A difference between transverse fields and longitudinal fields of great practical importance is that laser fields, once generated, propagate indefinitely in vacuum without the need for sources, whereas electric fields require sources to sustain them. The consequence in a typical laboratory laser experiment is that the beam that arrives at the target can only be a superposition of transverse fields. Any longitudinal fields that may arise from imperfections in optical elements such as gratings or mirrors will decay within a few wavelengths from the optical element.

Two phenomena will be discussed below to examine the limitations of the tunneling model. One is the nowfamiliar matter of laser-induced ionization. The other is electron-positron pair production from the vacuum without the intervention of any particle with mass. This subject has not been adequately examined, an assessment that applies to theory as well as physical interpretation of experiment.

The title of this article: "No light at the end of the tunnel" is based on the metaphor: "There is light at the end of the tunnel", implying that there is hope for the resolution of difficulties. That is negated here, with the significance that light does not create a quantum tunnel. 
Gaussian units are used for electromagnetic quantities. The terms "propagating field", "plane-wave field", "transverse field", "sourceless field", "laser field", and "light" are treated as equivalent.

\section{Laser-induced ionization}

Limitations of the tunneling model for strong-field atomic ionization have been discussed in Refs. [4-6]. The essence of the problem is most simply explicated in terms of the limit as the frequency of the laser field approaches zero. The DA, as employed in AMO physics, posits the complete absence of any magnetic component of the laser field. The low-frequency limit of an oscillatory electric field is simply a constant electric field, and the approach to that limit is referred to as adiabaticity. For many years, analytical approximations were judged defective unless they approached constant-field behavior as the frequency declined [8] - a false goal for laser interactions.

Laser fields propagate at the speed of light in vacuum independently of the frequency of the field. There can be no such phenomenon as a static limit of a propagating field. Some interesting extremely low-frequency examples of propagating fields are known that bear no resemblance to static fields. The Schumann resonance [9] is a phenomenon in which powerful lightning strikes generate radio waves that resonate in the cavity formed by the Earth's surface and the ionosphere. The fundamental frequency is $7.83 \mathrm{~Hz}$, corresponding to a wavelength about equal to the circumference of the Earth. Another example comes from the ability of several nations to communicate with deeply submerged submarines at great distances from the transmitter. The system used by the US Navy operated at $76 \mathrm{~Hz}^{-}$ with a wavelength of about one third Earth radius-and could communicate with deeply submerged submarines at locations halfway around the Earth [10].

The contrast between these applications of extremely low-frequency propagating fields and low-frequency longitudinal fields is striking. A low-frequency oscillatory electric field cannot propagate around the Earth, and it cannot penetrate a conducting medium like seawater. No information gleaned from the behavior of lowfrequency oscillatory electric fields has any relevance to the behavior of low-frequency propagating fields, even if the frequencies are the same.

As frequencies decline or as intensities increase, the behavior of propagating fields becomes relativistic. When the existence of a propagating field becomes the dominant influence in an interaction, then propagation at light speed means that the problem is relativistic. The behaviors of the radio waves in the Schumann resonance and the submarine communication application cited above are relativistic; they are not quasistatic electric.

Laser fields and oscillatory electric fields are fundamentally different phenomena. The tunneling model applies only to electric fields, not to plane-wave fields like those of lasers.

\section{Pairs from the vacuum}

Two mechanisms for the production of particleantiparticle pairs from the vacuum solely by electromagnetic means are the so-called "polarization of the vacuum" by an electric field, and pairs produced by the collision of photon beams.

Pairs produced by the collision of photon beams was first examined by Breit and Wheeler [11], so this mechanism for the production of pairs is called the BreitWheeler (BW) process.

Polarization of the vacuum was examined by Sauter [12], Heisenberg and Euler [13], and Schwinger [14]. The strength of electric field necessary to produce pairs from the vacuum is often called the "Schwinger limit," but it is identical to that found by Sauter. It is now common practice to refer to polarization of the vacuum as Sauter-Schwinger (SS) pair production.

\subsection{BW pair production}

The study of strong-field BW pair production revealed fundamental information about the distinction between strong-field electrodynamics and standard QED. These distinctions have no parallel with electric fields. Some of those results have not been fully incorporated into the literature on strong fields. They are summarized below.

\subsubsection{BW pairs in perturbation theory}

The lowest-order Feynman diagram for electronpositron pairs produced by photon-photon collision is a simple two-vertex diagram calculated by Breit and Wheeler in 1934 [11]. It is related to the other two-vertex phenomena involving electron and photon interactions: Compton scattering and pair annihilation. Although these processes are analytically similar, energy conservation requirements are very different. Pair annihilation is spontaneous and releases at least $2 m c^{2}$ of energy, whereas pair creation requires at least $2 m c^{2}$ of energy input from colliding photons.

\subsubsection{BW pairs in strong-field theory}

Vacuum pair production in which an intense photon beam collides with a counter-propagating energetic photon was calculated $[7,15]$ in what was initially a formal investigation of the analytical properties of QED. It has long been known that QED has a zero radius of convergence [16] despite the fact that a perturbation expansion shows remarkable accuracy when compared with experiment. The question explored was whether this convergence failure persisted in a theory based on a photon field sufficiently intense that it can be represented as an external (i.e., non-depletable) field. The 
method employed was based on the Volkov solution [17], an exact solution of the Dirac equation for a charged particle in an external photon field.

\subsubsection{Strong-field coupling parameter}

The strong-field BW investigation revealed that the coupling between an electron and a propagating electromagnetic field differs from that of ordinary QED. The coupling parameter for QED is the fine-structure constant $\alpha=e^{2} / \hbar c$. For the strong-field case, it is instead the intensity-dependent quantity [7]

$$
z_{f}=2 U_{p} / m c^{2}
$$

where $U_{p}$ is the ponderomotive potential of the electron in the field, defined in terms of the four-vector potential $A^{\mu}$ as

$$
U_{p}=\frac{e^{2}}{2 m c^{2}}\left\langle\left|A^{\mu} A_{\mu}\right|\right\rangle
$$

The angle brackets denote a cycle average and the absolute value is taken because $A^{\mu}$ is a space-like four vector. An alternative form for $z_{f}$ that provides useful insight follows if it is expressed as a multiple of $\alpha$ [18]

$$
z_{f}=\alpha \rho\left(2 \lambda t_{C}^{2}\right) .
$$

The quantity $\rho$ is the density of photons, $\lambda$ is the wavelength of the propagating field, and $\lambda_{C}=\hbar / m c$ is the electron Compton wavelength. Equation (3) thus states that with strong-field coupling, the fine-structure constant is multiplied by the number of photons contained within the volume $2 \lambda t_{C}^{2}$, which is essentially the volume of a cylinder of radius $\lambda_{C}$ and length $\lambda$. The Compton wavelength is the characteristic interaction length for a free electron, so it is not surprising to find this to be the radius of the interaction volume, but $\lambda$ can be a macroscopic quantity. The strong-field coupling parameter thus represents a bridge between QED and classical electrodynamics.

The fundamental coupling constant $z_{f}$ is equivalent to a parameter $\eta^{2}$ found in the literature on relativistic strong fields. The quantity $\eta$ is linearly dependent on the vector potential, and this dependence is frequently stated as $E / \omega$, with the apparent goal of introducing direct dependence on the electric field, assumed to be the quantity of paramount importance even though it comes with the further necessity of specifying the frequency $\omega$. This is inappropriate, and furthermore disguises the basic role of $z_{f}$.

The identification of a nonperturbative strong-field coupling constant is specific to propagating fields; it does not apply to static or oscillatory electric fields.

\subsubsection{Strong-field mass shell}

The relativistic energy-momentum relation

$$
p^{\mu} p_{\mu}=\frac{E^{2}}{c^{2}}-\mathbf{p}^{2}=(m c)^{2}
$$

is often called the "mass shell" of the electron. A matter of great practical and theoretical importance is that Eq. (4) is altered by strong fields to

$$
p^{\mu} p_{\mu}=(m c)^{2}\left(1+z_{f}\right)=(m c)^{2}+2 m U_{p} .
$$

This unanticipated consequence of strong fields was discovered independently by Sengupta [19] and by the present author $[7,15]$, and has been corroborated subsequently by many investigators. The term $2 m U_{p}$ has caused puzzlement, but it corresponds to a simple physical picture [18]. In a strong field, an electron acts as if it acquires a cloud of photons with energy $U_{p}$ and momentum $U_{p} / c$. The field-dressed electron thus becomes a particle with four momentum

$$
\Pi^{\mu}=p^{\mu}+\frac{1}{c} U_{p} \widehat{k}^{\mu},
$$

where $\widehat{k}^{\mu}$ is a unit four-vector on the light cone, since the component introduced by the entrained photons is on the light cone. The mass shell expression for the field-enhanced electron is

$$
\Pi^{\mu} \Pi_{\mu}=p^{\mu} p_{\mu}+\frac{2}{c} U_{p} p^{\mu} \widehat{k}_{\mu} .
$$

A four-vector on the mass shell is self-orthogonal, so $\widehat{k}^{\mu} \widehat{k}_{\mu}=0$, and $p^{\mu} \widehat{k}_{\mu}$ is a Lorentz invariant that can be evaluated in the rest frame of the electron, so that Eq. (7) becomes

$$
\Pi^{\mu} \Pi_{\mu}=(m c)^{2}+2 m U_{p} .
$$

The mass shell for a bare electron is given by Eq. (4), and the dressed electron is described by Eq. (8).

The identification of a modified mass shell is specific to propagating fields; it is not relevant for static or oscillatory electric fields.

The rendering of $z_{f}$ as a dimensionless form of $U_{p}$ for relativistic problems has counterparts in AMO phenomena as $[20,21]$

$$
z=U_{p} / \hbar \omega \text { and } z_{1}=2 U_{p} / E_{B},
$$

where $E_{B}$ is the binding energy of an electron in an atom or molecule. These parameters also apply to bandgap solids in strong fields [22].

\subsubsection{Channel closing}

In order for an electron participating in a strong-field process like pair production to satisfy four-momentum 
conservation, threshold conditions are modified because the electron and positron must each possess the entrained cloud of photons discussed above. This increases the required inputs from the colliding photon beams. If $N$ photons are the threshold number required to provide enough energy to produce pairs with bare electrons, there will be a field-induced increase to $N+1$ as the laser becomes more intense. Further indexing upward occurs as intensity increases. This is called "channel closing", first noted in strong-field atomic processes [20,23].

The concept of channel closing could occur also with oscillatory electric fields, since such a field induces a "quiver" kinetic energy equal to the ponderomotive potential energy that exists with plane-wave fields. That is, the cause of channel closing is a kinetic energy in one case and a potential energy in the other. However, in view of the lack of sufficiently strong oscillatory electric fields, no electric-field channel closing has been reported. It is routinely observed with laser fields.

\subsubsection{Convergence}

The initial stimulus for the study of strong-field BW pair production was to see if the Dyson demonstration [16] of a zero radius of convergence for QED applied as well when the laser field was treated as an external field. The answer is a qualified "no". The strong-field theory will have a convergent series expansion up to an intensity-limited value $[7,20]$. The limit can be stated in physical terms: when field intensity causes the first channel closing, this is a sufficient condition for the failure of a perturbation expansion. However, channel closing has not been shown to be a necessary condition; convergence might fail at an intensity less than that for the first channel closing.

\subsection{Laboratory observation of BW pair production}

A theoretical study in 1971 [24] examined the possibility of observing BW pair production using an energetic photon generated by the electron beam at the Stanford Linear Accelerator Center (SLAC) in collision with an intense laser beam. The necessary parameters to achieve success became available for a successful experiment at SLAC, reported in 1997 [25].

\subsubsection{Theoretical proposal}

When it became clear that high-powered lasers were in the offing, the formalism of Ref. [7] was applied to the practical goal of creating pairs from the vacuum in a laboratory setting. The primary significance of a realization of the proposal was stated in the title: "Production of electron pairs from a zero-mass state". The creation of energy by the diminution of mass was already a familiar process, but the creation of mass from pure energy had never been observed.

Several other "firsts" were also predicted. The experiment could prove the failure of perturbation theory, and even in perturbative terms, the lowest-order process allowed by conservation conditions would constitute the highest-order multiphoton process ever observed. (This was before the historic atomic physics experiment of Agostini, et al. [26].) It would also be a possible demonstration of the replacement of the mass shell of Eq. (4) by that of Eq. (5) [27].

The proposed experiment was to collide a laser beam with an energetic bremsstrahlung photon available as a by-product of the electron beam at SLAC. The calculation showed that the outcome would depend on the intensity of the laser, but not on its frequency. A major result of the calculation was the demonstration that a laser focal intensity of several times $10^{18} \mathrm{~W} / \mathrm{cm}^{2}$ would be required, even though there is no explicit threshold condition to govern that restriction. The 1971 calculated example, using a laser with a wavelength of $1060 \mathrm{~nm}$ and a peak SLAC electron beam energy of $18 \mathrm{Gev}$, requires a minimum laser photon order of $n_{0}=25$. When the same calculation is re-done for the parameters of the actual 1997 experiment with a $527 \mathrm{~nm}$ laser and $46.6 \mathrm{Gev}$ electron beam, the onset intensity is only slightly different even though the minimum laser photon order is reduced to $n_{0}=5$.

\subsubsection{SLAC experiment}

The pair production experiment conducted at SLAC $[25]$ is historic. It is the first and-so far-only experiment to produce electron pairs from the vacuum.

The experiment differed from the theoretical proposal only in that the energetic bremsstrahlung photon was replaced by an energetic photon produced by Compton back-scattering of laser photons from the SLAC electron beam. In view of this close correspondence, some of the descriptions employed in the landmark paper [25] are curious, and require comment.

The epochal achievement of creating mass from pure energy inexplicably is not mentioned.

The events observed are described in the article as "inelastic photon-photon scattering". However, the process can only be described as "scattering" if scattered photons are observed. There are no scattered photons detected in the experiment. Photon-photon scattering is one of the standard processes described in texts as a perturbative interaction of fourth-order. It is far less probable than the second-order pair production process, and no photon-photon scattering event could be observed with current technology. Light-bylight scattering has been reported [28] at the Large Hadron Collider, but only with the intervention of heavy-particle influences.

The observation of a nonperturbatively strong interaction in the SLAC experiment has been muddled because of the statement that they observe a multiphoton BW process with $n \geq 4$, but then show a power-law fit to their data with $n=5$. The perturbative mass shell of Eq. (4) predicts $n_{0}=4$, and the strong-field mass shell of Eq. (5) predicts $n_{0}=5$. If the process is truly perturbative, as is claimed, the lowest order $n_{0}=4$ 
would dominate. The measurement of $n_{0}=5$ is the evidence needed to demonstrate the failure of perturbation theory. This fundamental result demonstrated by the SLAC experiment is not mentioned [25].

\subsection{SS pair production}

The concept of SS pair production [12-14] is simple. In a particle-hole picture of electron states, electrons can occupy states with energies $\geq m c^{2}$, and positrons are viewed as negative-energy electrons with energies $\leq-m c^{2}$. The presence of a constant or quasistatic electric field tilts those boundaries, making it possible for a negative-energy electron to tunnel through to positive energies. The characteristic interaction length for a free electron is the Compton wavelength $\lambda=\hbar / m c$, and when half the distance between the entrance and exit of the tunnel is reduced to that length, this is regarded as the threshold for SS pair production. The required electric field is

$$
E=m^{2} c^{3} / e \hbar=1.3 \times 10^{18} \mathrm{~V} / m=\alpha^{-3} a . u .
$$

SS pairs, in principle, can be produced by oscillatory electric fields [29].

In practical terms, there is no known source of electric fields anywhere close to the magnitude shown in Eq. (10). The requirement for a vacuum free of objects with mass excludes the extreme Coulomb fields close to heavy nuclei.

The problem of creating such extreme electric fields has been discussed by many authors in the context of the electric component of powerful laser fields. There appears to have been no critical analysis of the association of the electric component of the transverse laser field with the oscillatory longitudinal electric field necessary to cause a tunneling phenomenon. The discussion above of the completely unrelated behaviors of the two type of fields that exists at high intensities or low frequencies leads to the conclusion that is inappropriate to associate the electric component of a laser field with the Sauter-Schwinger limit of Eq. (10).

An illustration of the disconnect between electric fields and propagating fields is supplied by the SLAC experiment [25]. The $527 \mathrm{~nm}$ laser field in collision with a photon of energy $29.2 \mathrm{GeV}$ produced electronpositron pairs at a field intensity of about $1.3 \times$ $10^{18} \mathrm{~W} / \mathrm{cm}^{2}$, with an electric component of $3 \times 10^{12} \mathrm{~V} / \mathrm{m}$, many orders of magnitude removed from the SS requirement of $10^{18} \mathrm{~V} / \mathrm{m}$.

A more universal result might be the electric field as measured in the center-of-momentum (c.m.) frame where $N$ laser photons of frequency $\omega_{c . m}$. match the momentum of the energetic photon of frequency $\widetilde{\omega}_{c . m}$ :

$$
N \hbar \omega_{c . m .} / c=\hbar \widetilde{\omega}_{c . m .} / c .
$$

The c.m. quantities can be found from the knowledge that the product $\left(N \hbar \omega_{c . m .}\right)\left(\hbar \widetilde{\omega}_{c . m}\right.$. $)$ is invariant under
Lorentz transformations along the direction of propagation $[7,24]$. This product can be given a specific value from the threshold condition

$$
\left(N \hbar \omega_{c m}\right)\left(\hbar \widetilde{\omega}_{c m}\right) \geq\left(m c^{2}\right)^{2}\left(1+z_{f}\right) .
$$

Equations (11) and (12), evaluated with the SLAC parameters $N=5, z_{f}=0.13$ yield the result that the laser electric field Lorentz transforms to $10^{17} \mathrm{~V} / \mathrm{m}$ in the c.m. system. This is an order of magnitude less than the Sauter-Schwinger limit, and depends upon the quantities $\omega, \widetilde{\omega}, N$. These will change with the laboratory setup. There is no fixed order of magnitude for the electric field associated with laser-induced pair creation.

Discussion of the Sauter-Schwinger limit in terms of the electric component of a laser field is a non sequitur; purely electric phenomena and propagating-field phenomena in strong fields are unrelated.

\section{Summary}

Electric fields couple to charged particles through the purely electric quantity $q E$. The coupling to charged particles of laser fields, as for all transverse fields, is measured by the ponderomotive potential $U_{p}$, which can be rendered in dimensionless form as $z_{f}=$ $2 U_{p} / m c^{2}$. Quantum tunneling through a potential barrier is exclusively an electric-field phenomenon that can serve as an approximation for laser-induced processes only over a limited range of parameters, and fails completely at high intensities and/or low frequencies. That failure of correspondence between longitudinal and transverse fields becomes extreme under conditions that enable production of electron-positron pairs from the vacuum. The sole experimental observation of laserproduced creation of matter from a zero-mass initial state (i.e., Breit-Wheeler pair production) overlooked important features of the experiment. Assessment of the electric field component of a laser field in terms of conditions necessary for achievement of Sauter-Schwinger pair production is improper.

Funding Open Access funding enabled and organized by Projekt DEAL.

Data Availability Statement This manuscript has no associated data or the data will not be deposited. [Authors' comment: Failure of the DA can be based on the magnitude of radiation-pressure displacement in the propagation direction during a wave period. When this is as large as one a.u., DA failure is extreme, and quantitative effects can be detected at displacements of $1 / 40$ a.u. as in experiments at $1.5 \mu \mathrm{m}$. This information about limits on applicability of the DA is illustrated in Fig. 2 of Ref. [4], based on the displacement measure $\beta_{0}$ shown in Eq. (5) in Ref. [5]. Unpublished results from Ohio State University (my thanks to Prof. Pierre Agostini) show effects that are nearly relativistic in experiments at $3.5 \mu \mathrm{m}$.]. 
Open Access This article is licensed under a Creative Commons Attribution 4.0 International License, which permits use, sharing, adaptation, distribution and reproduction in any medium or format, as long as you give appropriate credit to the original author(s) and the source, provide a link to the Creative Commons licence, and indicate if changes were made. The images or other third party material in this article are included in the article's Creative Commons licence, unless indicated otherwise in a credit line to the material. If material is not included in the article's Creative Commons licence and your intended use is not permitted by statutory regulation or exceeds the permitted use, you will need to obtain permission directly from the copyright holder. To view a copy of this licence, visit http://creativecomm ons.org/licenses/by/4.0/.

\section{References}

1. G. Gamow, Z. Phys. 51, 204 (1928)

2. J.R. Oppenheimer, Phys. Rev. 31, 66 (1928)

3. H.R. Reiss, Phys. Rev. A 100, 052105 (2019)

4. H.R. Reiss, Phys. Rev. Lett. 101, 043002 (2008)

5. H.R. Reiss, Phys. Rev. Lett. 101, 159901(E) (2008)

6. H.R. Reiss, J. Phys. B 47, 204006 (2014)

7. H.R. Reiss, J. Math. Phys. 3, 59 (1962)

8. C.J. Joachain, N.J. Kylstra, and R.M. Potvliege (Cambridge Univ. Press, Cambridge, 2012)

9. W.O. Schumann, Z. Naturforsch A 7, 250 (1952)

10. http://en.wikipedia.org/wiki/Project_Sanguine

11. G. Breit, J.A. Wheeler, Phys. Rev. 46, 1087 (1934)

12. F. Sauter, Z. Phys. 69, 742 (1931)

13. W. Heisenberg, H. Euler, Z. Phys. 98, 714 (1936)
14. J. Schwinger, Phys. Rev. 82, 664 (1951)

15. H.R. Reiss, Ph.D. thesis, University of Maryland, (1958)

16. F.J. Dyson, Phys. Rev. 85, 631 (1952)

17. D.M. Volkov, Z. Phys. 94, 250 (1935)

18. H.R. Reiss, Phys. Rev. A 89, 022116 (2014)

19. N.D. Sengupta, Bull. Math. Soc. (Calcutta) 44, 175 (1952)

20. H.R. Reiss, Phys. Rev. A 22, 1786 (1980)

21. H.R. Reiss, Prog. Quant. Electr. 16, 1 (1992)

22. H.D. Jones, H.R. Reiss, Phys. Rev. B 16, 2466 (1977)

23. H.J. Muller, A. Tip, M.J. van der Wiel, J. Phys. B 16, L679 (1983)

24. H.R. Reiss, Phys. Rev. Lett. 26, 1072 (1971)

25. D.L. Burke et al., Phys. Rev. Lett. 79, 1626 (1997)

26. P. Agostini, F. Fabre, G. Mainfray, G. Petite, N.K. Rahman, Phys. Rev. Lett. 42, 1127 (1979)

27. H.R. Reiss, Phys. Rev. D 6, 385 (1972)

28. D. d'Enterria, G.G. da Silveira, Phys. Rev. Lett. 111, 080405 (2013)

29. E. Brezin, C. Itzykson, Phys. Rev. D 2, 1191 (1970) 